\title{
Ultralow-emittance measurement of high-quality electron beams from a laser wakefield accelerator
}

\author{
Zhiyong Qin ${ }^{1,2}$, Changhai Yu1,a), Wentao Wang ${ }^{1, b)}$, Jiansheng Liu' ${ }^{1,3, c)}$, Wentao \\ $\mathrm{Li}^{1,4}$, Rong Qi ${ }^{1}$, Zhijun Zhang ${ }^{1}$, Jiaqi Liu' ${ }^{1,2}$, Ming Fang ${ }^{1,2}$, Ke Feng ${ }^{1,2}$, Ying $\mathrm{Wu}^{1,2}$, \\ Lintong $\mathrm{Ke}^{1,2}$, Yu Chen ${ }^{1,2}$, Yi Xu ${ }^{1,2}$, Yuxin Leng ${ }^{1,2}$, Cheng Wang ${ }^{1,2}$, Ruxin $\mathrm{Li}^{1,3,5}$, \\ and Zhizhan $\mathrm{Xu}^{1,3,5}$ \\ ${ }^{I}$ State Key Laboratory of High Field Laser Physics, Shanghai Institute of Optics and Fine Mechanics, Chinese Academy \\ of Sciences, Shanghai 201800, China \\ ${ }^{2}$ University of Chinese Academy of Sciences, Beijing 100049, China \\ ${ }^{3}$ Collaborative Innovation Center of IFSA (CICIFSA), Shanghai Jiao Tong University, Shanghai 200240, China \\ ${ }^{4}$ Department of Physics, SUPA and University of Strathclyde, Glasgow G4 0NG, United Kingdom \\ ${ }^{5}$ School of Physics Science and Technology, ShanghaiTech University, Shanghai 200031, China
}

By designing a cascaded laser wakefield accelerator, high-quality monoenergetic electron beams $(e$ beams) with peak energies of 340-360 MeV and $\mathrm{rms}$ divergence of $<0.3 \mathrm{mrad}$ were produced. Based on this accelerator, the e-beam betatron radiation spectra were measured exactly via the single-photon counting technique to diagnose the $e$-beam transverse emittance in a single shot. The $e$-beam transverse size in the wakefield was estimated to be $\sim 0.35 \mu \mathrm{m}$ by comparing the measured $\mathrm{x}$-ray spectra with the analytical model of synchrotron-like radiation. By combining the measured $e$-beam energy and divergence, the normalized transverse emittance was estimated to be as low as $56 \mu \mathrm{m} \mathrm{mrad}$ and consistent with particle-in-cell simulations. These high-energy ultralow-emittance $e$ beams hold great potential applications in developing free electron lasers and high-energy x-ray and gamma ray sources.

\section{INTRODUCTION}

The past decade has witnessed a remarkable progress in developing laser wakefield accelerators (LWFAs) ${ }^{1}$, which have already produced quasi-monoenergetic, low-emittance, GeV-class femtosecond $e$ beams by optimizing the injection method $^{2-10}$ and laser guiding techniques ${ }^{11-13}$. Compared with conventional radio-frequency linear accelerators, its ultrahigh accelerating gradient $(\sim 100 \mathrm{GV} / \mathrm{m})^{1}$ makes LWFA a very attractive compact accelerator ${ }^{10,13}$, holding great potential applications in the fields of high energy colliders; $\mathrm{THz}^{14,15}$, x-ray $^{16-19}$, and free-electron lasers $(\mathrm{FEL})^{20} ; \gamma$-ray radiation ${ }^{19,21,22}$; etc. In the

\footnotetext{
a)yuchanghai@siom.ac.cn

b)wwt1980@ siom.ac.cn

c) michaeljs_liu@siom.ac.cn
} 
blowout ${ }^{23}$ or "bubble" 24 regimes of the wakefield, electrons from the tail can be trapped into the bubble and accelerated to high energy longitudinally, while simultaneously oscillating transversely to emit x-rays due to the focusing forces of the bubble, known as betatron radiation ${ }^{19}$. Previous experiments and theories ${ }^{16,17,19}$ have demonstrated that the spectral characteristics of betatron radiation are similar to those of synchrotron radiation ${ }^{25}$ and typically determined by the betatron strength parameter, $K_{\beta}=\gamma \omega_{\beta} r_{b} / c$, where $r_{b}$ is the electron oscillation amplitude and can be referred as the e-beam transverse size, and $\gamma$ the relativistic factor of the electron, while $\omega_{\beta}=\omega_{p} / \sqrt{2 \gamma}$ and $\omega_{p}$ are the betatron oscillation frequency and plasma frequency, respectively. Because the betatron radiation spectra are strongly related to the $e$ beam transverse oscillation in the wakefield, and dominated by the high-energy part of the trajectory, the $e$-beam transverse size can be obtained by investigating the betatron radiation spectra without destructing the $e$ beam $^{19}$.

To realize the practical applications of LWFAs ${ }^{19,20,26}$, low-emittance $e$ beams ${ }^{27,28}$ are required specially to minimize the degradation over long-distance propagation. The transverse emittance, a nontrivial experimental parameter of high-energy $e$ beams, needs to be measured. A few methods have been proposed to measure the transverse emittance, such as the multi-screen image approach ${ }^{29}$, quadrupole $\operatorname{scan}^{30}$, "pepper-pot" technique ${ }^{31,32}$, etc. However, the first two methods are based on multi-shots and need highly stable and low-charge $e$ beams. Hence, they are not suitable for the LWFA $e$ beams. The "pepper-pot" technique is a destructive detection method and prevents the diagnosis of the $e$-beam energy spectra. Besides, this method has a limited resolution of $\sim 1 \mathrm{~mm}$ mrad. In addition to the above methods, the betatron radiation spectra measurement is an alternative method to measure the transverse normalized emittance, as demonstrated in previous experiments ${ }^{16,33,34}$. By measuring the betatron radiation spectra, the $e$-beam transverse size can be obtained. When combined with the $e$-beam peak energy and divergence angle measured in a single shot, the transverse emittance can be estimated.

In this paper, we present a single-shot high-resolution measurement of the betatron x-ray radiation spectra to estimate the transverse emittance of LWFA $e$ beams. The emitted x-ray photons in the range 1$16 \mathrm{keV}$ were detected with an x-ray charge-coupled device (CCD) camera in the far field based on the single-photon-counting (SPC) technique ${ }^{35-37}$. Besides, the x-ray total photon number and spatial distribution could be measured with an $\mathrm{x}$-ray detection system (XRDS) ${ }^{22}$. Comparing the obtained betatron spectra with the analytical model of synchrotron radiation, the $e$-beam transverse size in the plasma can then be obtained and the corresponding normalized transverse emittance can be estimated. To the best of our knowledge, the measured emittance of LWFA $e$ beams is much smaller than previous experimental 
results $^{34}$.

\section{EXPERIMENTAL SETUP}

The experiments were performed at the Ti:sapphire chirped pulse amplification laser system with a repetition rate of $1 \mathrm{~Hz}$ at $\mathrm{SIOM}^{10,22}$. The experimental setup is shown in Fig. 1(a), where an 800-nm laser system delivers up to $200 \mathrm{TW}$ with a full width at half maximum (FWHM) pulse duration of $33 \mathrm{fs}$. The laser pulses are focused on a two-stage gas nozzle by a $f / 30$ off-axis parabolic mirror with a focal spot size of $32 \mu \mathrm{m}$ (FWHM) and on-target peak power of $120 \mathrm{TW}$ with $\sim 61 \%$ energy concentration. This corresponds to a peak intensity of $\sim 4.0 \times 10^{18} \mathrm{~W} / \mathrm{cm}^{2}$ and gives a normalized vector potential of $a_{0} \approx$ 1.6. The gas target contains a two-segment $(0.8 \mathrm{~mm}+3 \mathrm{~mm})$ helium gas jets, and the right wall of the first gas jet $(0.8 \mathrm{~mm})$ is inserted into the downstream of the second one ( $3 \mathrm{~mm}$ diameter), as shown in Fig. 1(a). The laser is focused at the entrance of the first gas jet. By setting the backing pressures of the two gas jets to 5-6 and 3-4 bar, respectively, and maintaining the right wall of the first gas get next to the entrance of the second one, a structured gas profile with a density bump is formed to realize a high-quality cascaded LWFA $^{10}$. A probe beam was split from the driving laser pulse and passed transversely through the gas target to diagnose the plasma density, and the results are shown in Fig. 1(b). The plasma densities in the two jets are $(1.1 \pm 0.1) \times 10^{19} \mathrm{~cm}^{-3}$ and $(7.0 \pm 0.5) \times 10^{18} \mathrm{~cm}^{-3}$, respectively, and the density bump is located $\sim 0.15 \mathrm{~mm}$ away from the exit of the first gas jet, with a gas flow overlap region of $\sim 400$ $\mu \mathrm{m}$ and a highest density of $(1.5 \pm 0.1) \times 10^{19} \mathrm{~cm}^{-3}$. The generated $e$ beams were dispersed by a $90-$ $\mathrm{cm}$ long tunable dipole electromagnet with a maximum magnetic field of $1.1 \mathrm{~T}$, and detected by a Lanex phosphor screen imaged on an intensified CCD camera, which was cross-calibrated by using a calibrated imaging plate to measure the $e$-beam charge ${ }^{10,38}$. Fig. 1(c) shows a typical electron energy spectrum with a peak energy of $360 \mathrm{MeV}$.

The on-axis x-ray betatron radiation emitted by the $e$ beams from the LWFA was detected by a 16bit back-illuminated x-ray CCD (PIXIS-XO:400B) with 1340×400 pixels of $20-\mu \mathrm{m}$ size. As shown in Fig. 1(a), the X-ray CCD camera, encased in lead shielding, was operated in a SPC mode to measure the $\mathrm{x}$-ray spectra between $\sim 30 \mathrm{eV}$ to $16 \mathrm{keV}$ with a high energy resolution of $\sim 26 \mathrm{eV}$. The spectrum of the detected photons can be reconstructed by calculating the intensity histogram of the CCD signal. For the SPC mode, it is of primary importance that the detected photon flux should be low enough. To satisfy the SPC condition $^{35,37}$, the $\mathrm{x}$-ray CCD was positioned downstream far away $(\sim 5.3 \mathrm{~m})$ from the gas jets exit, which leads to an average of less than 0.2 photons per pixel. Besides, an 8 - $\mu \mathrm{m}$-thick $\mathrm{Al}$ foil was inserted in front of the $\mathrm{x}$-ray CCD camera to block the residual laser pulse and low-energy photons below $2 \mathrm{keV}$. This low 
photon flux guaranteed the SPC mode and led to a high signal-to-noise ratio. We also inserted a 10-cmlong and 3-mm-diameter wolfram collimator $\sim 0.2 \mathrm{~m}$ away from the gas jets exit along the propagation axis to minimize the broad-divergence bremsstrahlung $\mathrm{x}$-ray background radiation onto the $\mathrm{x}$-ray $\mathrm{CCD}$ camera. The acceptance angle was $\sim 10 \mathrm{mrad}$, which could effectively block the bremsstrahlung radiation caused by the electrons with low energy and large divergence angle, but was large enough to avoid influencing the measurement of the high energy $e$-beam betatron x-ray radiation.

To measure the x-ray photon number and spatial radiation profile exactly, we designed an XRDS, containing a $\mathrm{Lu}_{2} \mathrm{SiO}_{4}$ (LSO)-crystal scintillator and a 16-bit CCD camera. The XRDS was placed $\sim 4.7 \mathrm{~m}$ downstream from the LWFA. The residual laser pulse and the low-energy radiation $(<2 \mathrm{keV})$ were blocked by a 300- $\mu$ m-thick beryllium window, as shown in Fig. 1(a). The transmitted x-rays struck the LSO-crystal scintillator to produce a fluorescence signal and was then detected by the CCD camera ${ }^{39}$.

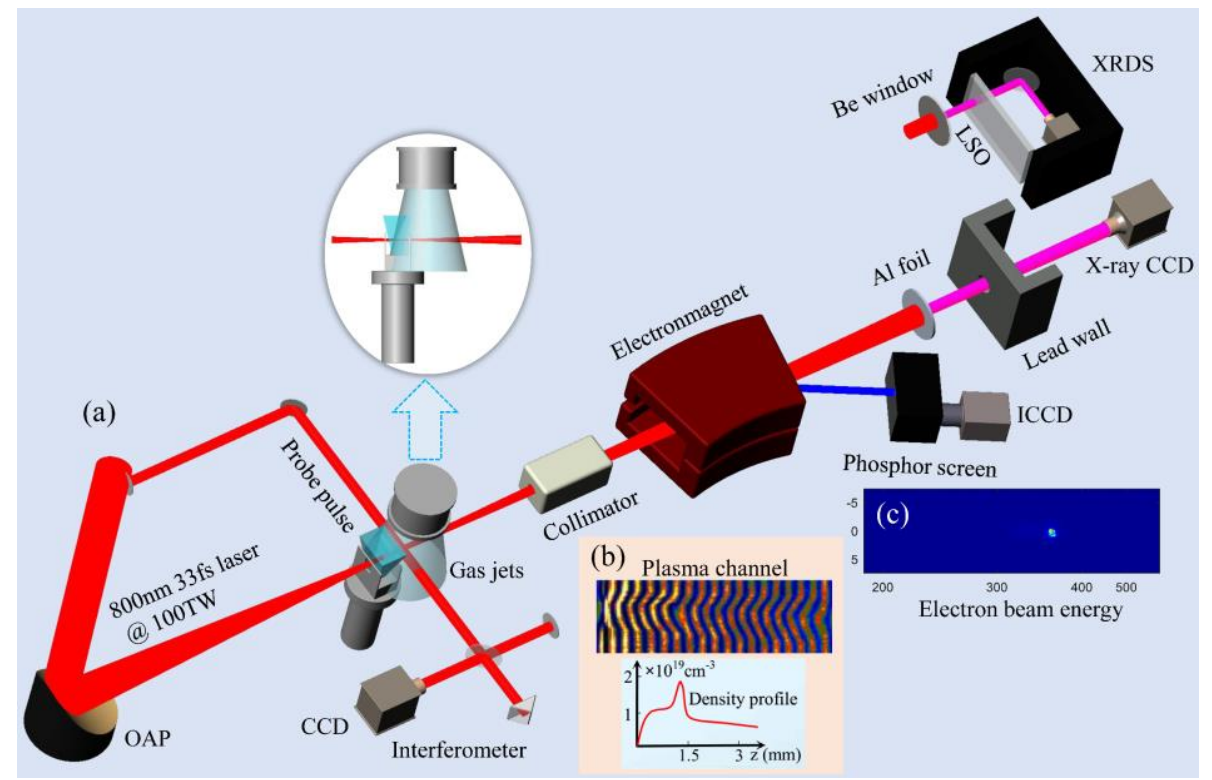

FIG.1. (Color Online) (a) Experimental setup of a LWFA for $e$-beam and betatron x-ray generation and diagnostics. (b) Measured plasma channel and plasma density profile via a Michelson-type interferometer. (c) Typical $e$-beam spectrum measured by the electromagnet.

\section{EXPERIMENTAL RESULTS AND DISCUSSION}

In our experiments, by adjusting the backing pressure of the gas jets and the distance of the two gas nozzles, quasi-monoenergetic $e$ beams with the peak energies in the range of 300 400 MeV were produced. The typical energy spectra and divergence angle of five shots under similar conditions are shown in Figs. 2(a)- 2(c). The peak energy, rms energy spread, rms divergence angle, and electron charges are listed in 
the table of Fig. 2(a). The peak energy is stable with an energy spread of $<1 \%$ and divergence angle of $<0.3 \mathrm{mrad}$. Moreover, to investigate the shot-to-shot fluctuations of the $e$ beams, a series of $e$ beam measurements over 60 shots were also recorded to analyze the fluctuation of the central positions, peak energy, and rms divergence angle of the $e$ beams, as shown in Figs. 2(d)-2(e). The statistics suggest that the $e$-beam energy was mainly located at $340-360 \mathrm{MeV}$ and the divergence angle varied from 0.15 to 0.4 mrad. Meanwhile, the maximum centroid offset of the $e$ beams is about $\pm 0.3 \mathrm{mrad}$, which can be attributed to the fluctuation of the laser pulse and density distribution.
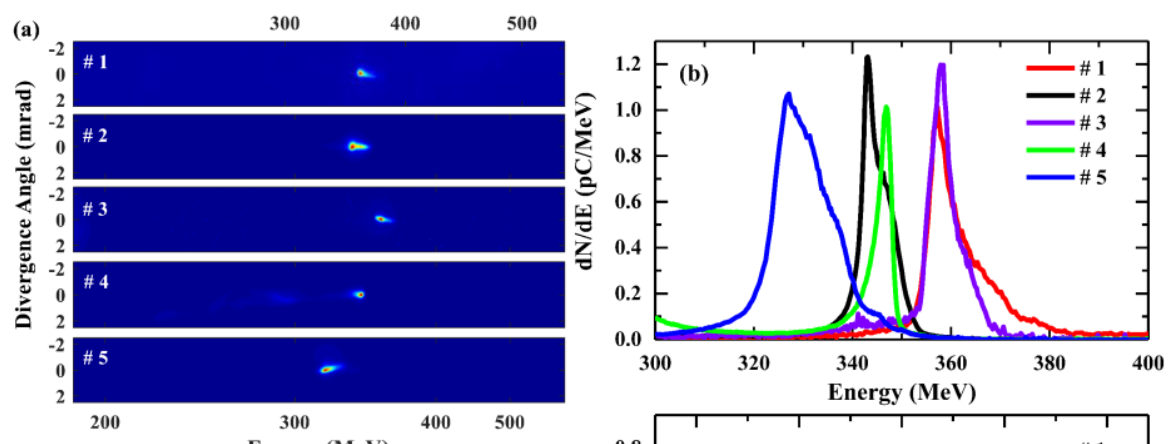

\begin{tabular}{|c|c|c|c|c|}
\hline & $\mathrm{E}(\mathrm{MeV})$ & $\mathrm{RMS} \Delta \mathrm{E} / \mathrm{E}$ & $\begin{array}{l}\mathrm{RMS} \sigma_{\theta} \\
(\mathrm{mrad})\end{array}$ & $\mathrm{Q}(\mathrm{pC})$ \\
\hline$\# 1$ & 357 & $0.75 \%$ & 0.23 & 12 \\
\hline$\# 2$ & 345 & $0.7 \%$ & 0.21 & 13.4 \\
\hline$\# 3$ & 358 & $0.59 \%$ & 0.14 & 12.8 \\
\hline$\# 4$ & 347 & $0.38 \%$ & 0.27 & 10.6 \\
\hline$\# 5$ & 327 & $1.27 \%$ & 0.2 & 15.4 \\
\hline
\end{tabular}


FIG. 2. (Color Online) (a) Typical e-beam energy distribution for five consecutive shots; (b-c) Corresponding $e$-beam energy spectra and rms divergence angle distributions; (d-e) Statistics of shot-toshot fluctuations of the $e$-beam central position and rms divergence angle over 60 shots.

To measure the betatron radiation, a single-shot x-ray radiation pattern corresponding to the $e$-beam energy spectra of shot \#1 in Fig. 2(a) was detected by using the x-ray CCD camera, as shown in Fig. 3(a), 
and the reconstructed x-ray spectral profile is presented in Fig. 3(c) by the red curve. Besides, the betatron $\mathrm{X}$-ray spectra can be characterized by a synchrotron-like on-axis spectrum ${ }^{25}$ of the form $d^{2} I /\left.d E d \Omega\right|_{\theta=0} \propto$ $\left(E / E_{c}\right)^{2} \mathcal{K}_{2 / 3}^{2}\left(E / E_{c}\right)$, where $\mathcal{K}_{2 / 3}$ is the second kind modified Bessel function of $2 / 3$ order. $E_{c}$ is the critical energy, which is given by $E_{c}=3 \hbar K_{\beta} \gamma^{2} \omega_{\beta}$ and $K_{\beta}=\gamma \omega_{\beta} r_{b} / c \approx 1.33 \times$ $10^{-10} r_{b}[\mathrm{um}] \sqrt{\gamma n_{e}\left[\mathrm{~cm}^{-3}\right]}$, where $n_{e}=7 \times 10^{18} \mathrm{~cm}^{-3}$ is the plasma density and the $e$-beam energy of shot \#1 in Fig. 2(a) is $357 \mathrm{MeV}(\gamma \sim 700)$. Since the betatron spectral shape is closely associated with the critical energy and $e$-beam transverse size, the measured betatron spectra are fitted under different critical energies by the synchrotron-like on-axis radiation form. Good agreement between the experimental spectra and the theoretical spectra, convolved with CCD camera and filter response, is obtained for the critical energy $E_{c}=6.2 \pm 0.5 \mathrm{keV}$, as shown in Fig. 3(c) with the blue curve. The light blue shadow defines the uncertainty. Since both the electron parameters and the x-ray betatron radiation were measured simultaneously in a single laser shot, they can be used to be verified mutually. According to the critical energy indicated in Fig. 3(c), the e-beam transverse size of shot \#1 in Fig. 2(a) can be estimated to be $r_{b} \approx 0.35 \pm 0.03 \mu \mathrm{m}$.

Because that the experimental spectra are integrated over the opening angle of the x-ray CCD camera, while the theoretical model only involves the on-axis portion. Therefore, to investigate the effect of the radiated angular distribution on the fitting result, we also simulated the integration spectra ${ }^{19,40,41}$ numerically over the emission angle of the synchrotron radiation produced by a single electron trajectory with an oscillation amplitude of $0.37 \mu \mathrm{m}$. The simulated angle-integrated spectra convolved with the CCD camera and filter response is shown as the green curve in Fig. 3(c), which is in good agreement with the on-axis spectra and the experimental spectra.

By using the $e$-beam transverse size of shot \#1 in Fig. 2(a), the betatron strength parameter was calculated to be $K_{\beta} \approx 3.25$, and the theoretical divergence angle of the betatron radiation can be estimated to be $\theta \approx K_{\beta} / \gamma \approx 4.65 \mathrm{mrad}$. Furthermore, the electron oscillation period number was estimated as $N_{0}=L / \lambda_{\beta} \approx 5$, where $L$ is the wiggler length and $\lambda_{\beta}=\sqrt{2 \gamma} \lambda_{p}$ the betatron wavelength. The total photon number could thus be predicted ${ }^{42}$ to be $\left\langle N_{p h}\right\rangle \simeq 5.6 \times 10^{-3} N_{0} N_{e} K_{\beta} \sim 1.0 \times 10^{7}$, where $N_{e}$ is the $e$-beam charge of shot \#1. To further verify the reasonableness of the transverse $e$-beam size, we also detected the betatron radiation pattern and total radiated photon number by the XRDS, as shown in Figs. 3(b), (d), and (e), which correspond to the e-beam spectra of shot \#3 in Fig. 2(a). The total photon number of the betatron radiation can be estimated to be $(1.2 \pm 0.2) \times 10^{7}$. Fig. 3(d) and 3(e) are the corresponding betatron radiation divergence angles in the horizontal ( $\mathrm{z}$ axis) and vertical (y axis) directions, respectively. 
It can be found that the radiation divergence angles were $4.0 \mathrm{mrad}$ and $2.5 \mathrm{mrad}$, respectively. Although only one of the two x-ray diagnostic systems could be installed in one shot, the betatron radiation of shot \#1 and shot \#3 could be assumed to be the same due to their identical acceleration energy. The measured total photon number and radiation divergence angle by XRDS are both almost consistent with the theoretical estimation based on the $0.35-\mu \mathrm{m} e$-beam transverse size. Therefore, we can infer that the $e$ beam transverse size of $r_{b} \approx 0.35 \pm 0.03 \mu \mathrm{m}$, achieved through the fitting to the betatron radiation spectrum, is reasonable.

By measuring the $e$-beam spectrum and the corresponding betatron x-ray radiation spectrum in a single laser shot, we have obtained the $e$-beam peak energy $(357 \mathrm{MeV})$, divergence angle $(0.23 \mathrm{mrad})$, and $e$ beam size $(0.35 \mu \mathrm{m})$. Therefore, the normalized transverse emittance can be estimated using $\epsilon_{x} \approx \gamma r_{b} \sigma_{\theta}$ to be $56 \pm 5 \mu \mathrm{m}$ mrad for shot \#1 in Fig. 2(a), which is the smallest value measured by experiments so far.


FIG. 3 (Color Online) (a) Background-subtracted x-ray beam recorded by an x-ray CCD camera in the SPC mode. (b) Betatron X-ray radiation pattern detected by the XRDS method. (c) Betatron X-ray radiation spectrum retrieved by the x-ray CCD camera in a single shot (red curve), fitted by the synchrotron on-axis radiation calculation (blue curve) and angle-integrated theoretical spectrum (green curve). (d)-(e) Gaussian fitting of the betatron radiation profile of (b) in the $\mathrm{z}$ and $\mathrm{y}$ directions, respectively.

\section{TWO-DIMENSIONAL PIC SIMULATIONS}


To better understand the experimental result, a two-dimensional particle-in-cell (2D PIC) simulation was performed with the code VORPAL ${ }^{43}$. A linearly polarized Gaussian laser pulse with wavelength $\lambda_{L}=$ $0.8 \mu \mathrm{m}$, normalized amplitude $a_{0}=1.6$, spot size $w_{0}=31 \mu \mathrm{m}(\mathrm{FWHM})$, and pulse duration $\tau_{L}=$ 35 fs (FWHM) was used in simulations. A moving window with a size of $75 \times 130 \mu \mathrm{m}^{2}$ was chosen and grid cell size was $\lambda_{L} / 25$ in the propagation direction and $\lambda_{L} / 12$ in the transverse direction. By choosing the plasma density close to the experimental condition, as shown in Fig. 4(a), a high-quality $e$ beam can be produced. Fig. 4(b)-(f) show snapshots of the simulated density distribution and wakefield structure at $\mathrm{x}=1.0(\mathrm{~b}), 1.5$ (c), 2.0 (d), 2.3 (e), and $3.0 \mathrm{~mm}(\mathrm{f})$, respectively, where $\mathrm{x}$ is the propagation direction. The $e$ beam was trapped into the second bucket at about $\mathrm{x}=0.9 \mathrm{~mm}$ by self-injection and being seeded into the first bucket for re-acceleration after the density bump. The evolution of the peak energy, rms energy spread, transverse size, and normalized transverse emittance of the $e$ beam along the propagation direction are all shown in Figs. 4(g)-(h). The peak energy of the simulated $e$ beam is $366 \mathrm{MeV}$ with an rms energy spread of $1.0 \%$, a transverse beam size of $0.4 \mu \mathrm{m}$, and a normalized transverse emittance of $60 \mu \mathrm{m} \mathrm{mrad}$, all these are in good agreement with experimental results. In the simulations, the self-injected $e$ beam experiences a quasi-stable acceleration and a negative energy chirp ${ }^{10,44}$ in the density bump upward and downward region, respectively, which leads to an e-beam energy spread compression during reacceleration in the first bucket. Therefore, a very low energy spread can be obtained. Moreover, from Fig. 4(b) it can be found that the $e$ beam is self-injected into the second bucket longitudinally, which leads to small transverse momentum and transverse position. This longitudinal self-injection mechanism will lead to a low transverse emittance in the acceleration process ${ }^{8,28}$. After being seeded into the base of the first bucket, the $e$ beam experiences the highest accelerating field and maintains a small transverse momentum for re-acceleration, as shown in Fig. 4(c-f). Thus, owing to the initial small transverse momentum in the second bucket and a proper seeding phase and energy spread compression in the first bucket, an ultralow normalized transverse emittance can be obtained. Therefore, by adjusting the backing pressures and the relative distance between the two nozzles, a high-quality $e$ beam with a low energy spread and an ultralow normalized transverse emittance can be produced and controlled ${ }^{45}$.

Furthermore, to investigate the betatron oscillation and x-ray radiation, we traced the trajectories of the sampled accelerating electrons in the plasma as shown in Fig. 4(h), and the corresponding betatron radiation spatial distribution is plotted in Fig. 4(i). The trajectory traced in Fig. 4(h) is typical for the accelerated electrons and the number of oscillations is about 5. The corresponding spatial distribution of radiation is calculated by some randomly sampled electrons, as shown in Fig. 4(i). It shows that the radiation divergence angles in the transverse direction are $4.2 \mathrm{mrad}$, which is agreement with the 
experimental estimation above. The on-axis radiation spectrum is shown in Fig. 4(i) as a red curve. Its critical radiation energy is about $6.3 \mathrm{keV}$, which is also consistent with experimental result. Therefore, it indicates that the bubble structure in the experiment is stable and the $e$-beam transverse oscillation is isotropic $^{46}$, which may contribute to a high quality $e$-beam generation.
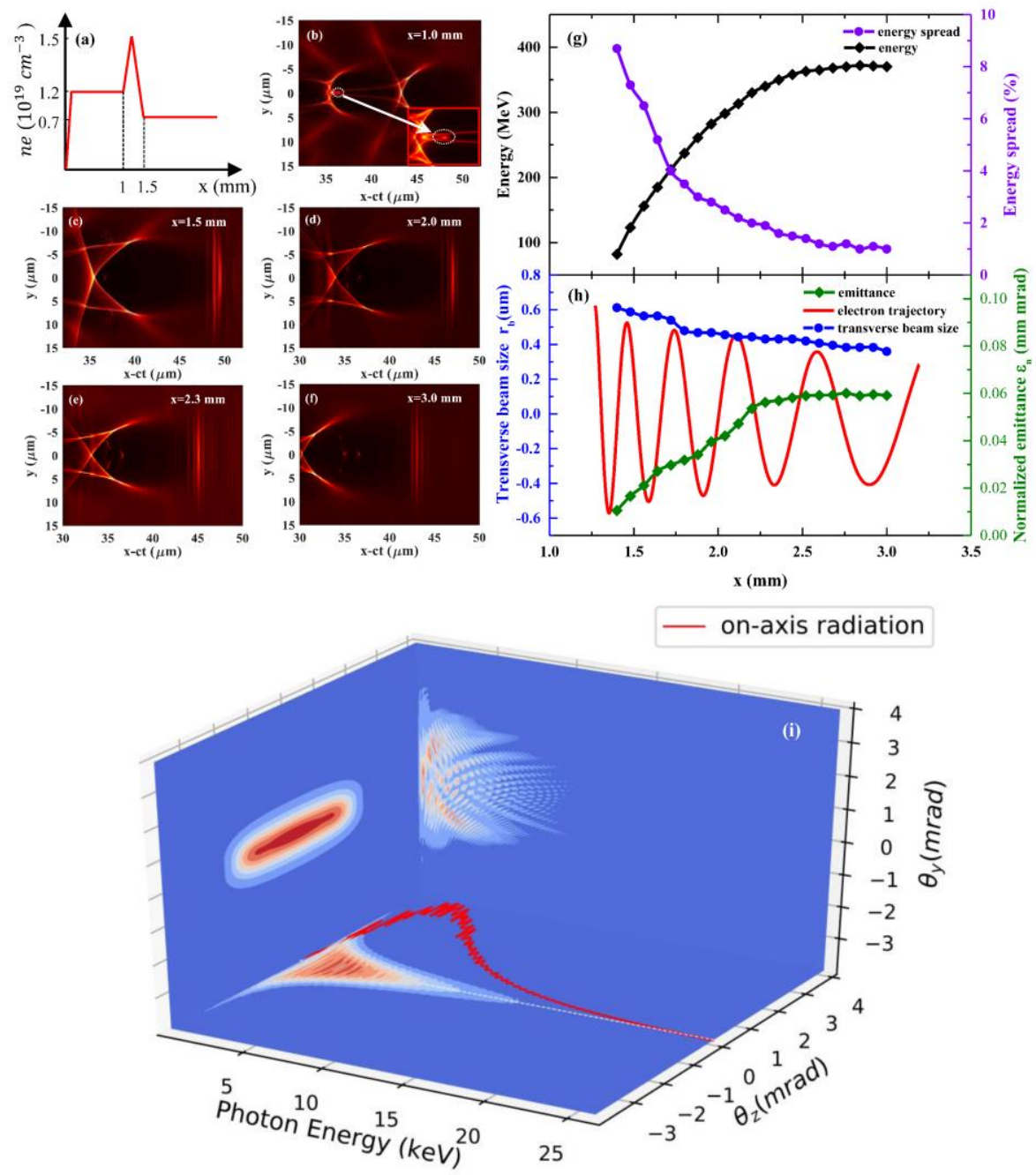

FIG. 4 (Color Online) (a) Longitudinal plasma density profile in simulations. (b)-(f) Plasma density distribution and wakefield structure in the 2D PIC simulation at x = 1.0 (b), 1.5 (c), 2.0 (d), 2.3 (e), and $3.0 \mathrm{~mm}$ (f), respectively. (g) Evolution of the e-beam peak energy (black curve) and rms energy spread (violet curve) in simulations. (h) Transverse beam size (blue curve) and the corresponding normalized transverse emittance (green curve) evolution and typical trajectory (red curve) of one of the accelerated electrons. (i) Spatial distribution in the transverse directions and radiation spectrum of the corresponding betatron radiation by some representative electrons during acceleration.

\section{CONCLUSIONS}


In conclusion, we have presented a single-shot high-resolution x-ray spectra characterization of betatron radiation in a cascaded LWFA. In this LWFA, stable monoenergetic $e$ beams have been produced and the measurement of the corresponding single-shot betatron x-ray spectra in the range of 2-20 keV was also enabled by an x-ray CCD camera in the SPC mode. Comparing the measured x-ray spectra with an analytical model of betatron radiation, the $e$-beam transverse size in the plasma was estimated to be $0.35 \pm 0.03 \mu \mathrm{m}$. Furthermore, the betatron radiation pattern and total radiated photon number were also measured by the XRDS method, and both measured results are in good agreement with the theoretical estimation. Combining the measured $e$-beam transverse size with its divergence angle of $0.23 \mathrm{mrad}$, a normalized transverse emittance as low as $56 \pm 5 \mu \mathrm{m}$ mrad was inferred for an $e$ beam with $357-\mathrm{MeV}$ peak energy, $0.75 \% \mathrm{rms}$ energy spread, and 12-pC charge. The peak energy and normalized emittance of the $e$ beam in the experiment are consistent with 2D PIC simulations. This ultralow emittance obtained in the experiment demonstrates the potential of this high-quality $e$ beam for x-ray FEL, bright gamma-ray sources, and colliders for high-energy physics.

\section{ACKNOWLEDGMENTS}

This work was supported by the National Natural Science Foundation of China (Grant Nos. 11127901, 11425418, 61521093, and 11505263) and the Strategic Priority Research Program (B) (Grant No. XDB16) of the State Key Laboratory Program of the Chinese Ministry of Science and Technology.

\section{Reference}

1 T. Tajima and J. M. Dawson, Phys. Rev. Lett. 43 (4), 267 (1979).

2 W. T. Wang, W. T. Li, J. S. Liu, Ch. Wang, Q. Chen, Z. J. Zhang, R. Qi, Y. X. Leng, X. Y. Liang, Y. Q. Liu, X. M. Lu, C. Wang, R.X. Li, and Z. Z. Xu, Appl. Phys. Lett. 103 (24), 243501 (2013).

3 A. Pak, K. A. Marsh, S. F. Martins, W. Lu, W. B. Mori, and C. Joshi, Phys. Rev. Lett. 104 (2), 025003 (2010).

4 C. McGuffey, A. G. R. Thomas, W. Schumaker, T. Matsuoka, V. Chvykov, F. J. Dollar, G. Kalintchenko, V. Yanovsky, A. Maksimchuk, K. Krushelnick, V. Yu Bychenkov, I. V. Glazyrin, and A. V. Karpeev, Phys. Rev. Lett. 104 (2), 025004 (2010).

5 K. Schmid, A. Buck, C. M. S. Sears, J. M. Mikhailova, R. Tautz, D. Herrmann, M. Geissler, F. Krausz, and L. Veisz, Phys. Rev. ST Accel. Beams 13 (9), 091301 (2010).

6 J. S. Liu, C. Q. Xia, W. T. Wang, H. Y. Lu, Ch Wang, A. H. Deng, W. T. Li, H. Zhang, X. Y. Liang, 
Y. X. Leng, X. M. Lu, C. Wang, J. Z. Wang, K. Nakajima, R. X. Li, and Z. Z. Xu, Phys. Rev. Lett. 107 (3), 035001 (2011).

B. B. Pollock, C. E. Clayton, J. E. Ralph, F. Albert, A. Davidson, L. Divol, C. Filip, S. H. Glenzer, K. Herpoldt, W. Lu, K. A. Marsh, J. Meinecke, W. B. Mori, A. Pak, T. C. Rensink, J. S. Ross, J. Shaw, G. R. Tynan, C. Joshi, and D. H. Froula, Phys. Rev. Lett. 107 (4), 045001 (2011).

S. Corde, C. Thaury, A. Lifschitz, G. Lambert, K. Ta Phuoc, X. Davoine, R. Lehe, D. Douillet, A. Rousse, and V. Malka, Nat. Commun 4, 1501 (2013).

R. Lehe, A. F. Lifschitz, X. Davoine, C. Thaury, and V. Malka, Phys. Rev. Lett. 111 (8), 085005 (2013).

W. T. Wang, W. T. Li, J. S. Liu, Z. J. Zhang, R. Qi, C. H. Yu, J. Q. Liu, M. Fang, Z. Y. Qin, C.Wang, Y. Xu, F. X. Wu, Y. X. Leng, R. X. Li, and Z. Z. Xu, Phys. Rev. Lett. 117 (12), 124801 (2016).

J. E. Ralph, K. A. Marsh, A. E. Pak, W. Lu, C. E. Clayton, F. Fang, W. B. Mori, and C. Joshi, Phys. Rev. Lett. 102 (17), 175003 (2009).

W. P. Leemans, B. Nagler, A. J. Gonsalves, Cs Toth, K. Nakamura, C. G. R. Geddes, E. Esarey, C. B. Schroeder, and S. M. Hooker, Nat. Phys 2 (10), 696 (2006).

W. P. Leemans, A. J. Gonsalves, H. S. Mao, K. Nakamura, C. Benedetti, C. B. Schroeder, C. Toth, J. Daniels, D. E. Mittelberger, S. S. Bulanov, J. L. Vay, C. G. Geddes, and E. Esarey, Phys. Rev. Lett. 113 (24), 245002 (2014).

W. P. Leemans, C. G. R. Geddes, J. Faure, Cs Tóth, J. van Tilborg, C. B. Schroeder, E. Esarey, G. Fubiani, D. Auerbach, B. Marcelis, M. A. Carnahan, R. A. Kaindl, J. Byrd, and M. C. Martin, Phys. Rev. Lett. 91 (7), 074802 (2003).

J. van Tilborg, C. B. Schroeder, C. V. Filip, Cs Tóth, C. G. R. Geddes, G. Fubiani, R. Huber, R. A. Kaindl, E. Esarey, and W. P. Leemans, Phys. Rev. Lett. 96 (1), 014801 (2006).

S. Kneip, C. McGuffey, J. L. Martins, S. F. Martins, C. Bellei, V. Chvykov, F. Dollar, R. Fonseca, C. Huntington, G. Kalintchenko, A. Maksimchuk, S. P. D. Mangles, T. Matsuoka, S. R. Nagel, C. A. J. Palmer, J. Schreiber, K. Ta Phuoc, A. G. R. Thomas, V. Yanovsky, L. O. Silva, K. Krushelnick, and Z. Najmudin, Nat. Phys 6 (12), 980 (2010).

L. M. Chen, W. C. Yan, D. Z. Li, Z. D. Hu, L. Zhang, W. M. Wang, N. Hafz, J. Y. Mao, K. Huang, Y. Ma, J. R. Zhao, J. L. Ma, Y. T. Li, X. Lu, Z. M. Sheng, Z. Y. Wei, J. Gao, and J. Zhang, Sci. Rep 3, 1912 (2013).

K. Huang, Y. F. Li, D. Z. Li, L. M. Chen, M. Z. Tao, Y. Ma, J. R. Zhao, M. H. Li, M. Chen, M. Mirzaie, N. Hafz, T. Sokollik, Z. M. Sheng, and J. Zhang, Sci. Rep 6, 27633 (2016).

S. Corde, K. Ta Phuoc, G. Lambert, R. Fitour, V. Malka, A. Rousse, A. Beck, and E. Lefebvre, Rev. Mod. Phys. 85 (1), 1 (2013).

Zhirong Huang, Yuantao Ding, and Carl B. Schroeder, Phys. Rev. Lett. 109 (20), 204801 (2012). 
K. Ta Phuoc, CordeS, ThauryC, MalkaV, TafziA, J. P. Goddet, R. C. Shah, SebbanS, and RousseA, Nat. Photon 6 (5), 308 (2012).

C. H. Yu, R. Qi, W. T. Wang, J. S. Liu, W. T. Li, C. Wang, Z. J. Zhang, J. Q. Liu, Z. Y. Qin, M. Fang, K. Feng, Y. Wu, Y. Tian, Y. Xu, F. X. Wu, Y. X. Leng, X. F. Weng, J. H. Wang, F. L. Wei, Y. C. Yi, Z. H. Song, R. X. Li, and Z. Z. Xu, Sci. Rep 6, 29518 (2016).

W. Lu, M. Tzoufras, C. Joshi, F. S. Tsung, W. B. Mori, J. Vieira, R. A. Fonseca, and L. O. Silva, Phys. Rev. ST Accel. Beams 10 (6), 061301 (2007).

A. Pukhov, S. Gordienko, S. Kiselev, and I. Kostyukov, Plasma Phys. Control. Fusion 46 (12B), B179 (2004).

E. Esarey, B. A. Shadwick, P. Catravas, and W. P. Leemans, Phys. Rev. E 65 (5), 056505 (2002).

E. Esarey, C. B. Schroeder, and W. P. Leemans, Rev. Mod. Phys. 81 (3), 1229 (2009).

Klaus Floettmann, Phys. Rev. ST Accel. Beams 6 (3), 034202 (2003).

M. Chen, E. Esarey, C. G R Geddes, E. Cormier-Michel, C. B Schroeder, S. S Bulanov, C. Benedetti, L. L Yu, S. Rykovanov, D. L Bruhwiler, and W. P Leemans, Phys. Rev. ST Accel. Beams 17 (5), 051303 (2014).

Vitaly Yakimenko, Marcus Babzien, Ilan Ben-Zvi, Robert Malone, and Xijie Wang, Nucl. Instrum. Methods Phys. Res., Sect. A 483 (1), 277 (2002).

R. Weingartner, S. Raith, A. Popp, S. Chou, J. Wenz, K. Khrennikov, M. Heigoldt, A. R. Maier, N. Kajumba, M. Fuchs, B. Zeitler, F. Krausz, S. Karsch, and F. Grüner, Phys. Rev. ST Accel. Beams 15 (11), 111302 (2012).

E. Brunetti, R. P. Shanks, G. G. Manahan, M. R. Islam, B. Ersfeld, M. P. Anania, S. Cipiccia, R. C. Issac, G. Raj, G. Vieux, G. H. Welsh, S. M. Wiggins, and D. A. Jaroszynski, Phys. Rev. Lett. 105 (21), 215007 (2010).

Christopher M. S. Sears, Alexander Buck, Karl Schmid, Julia Mikhailova, Ferenc Krausz, and Laszlo Veisz, Phys. Rev. ST Accel. Beams 13 (9), 092803 (2010).

S. Kneip, C. McGuffey, J. L. Martins, M. S. Bloom, V. Chvykov, F. Dollar, R. Fonseca, S. Jolly, G. Kalintchenko, K. Krushelnick, A. Maksimchuk, S. P. D. Mangles, Z. Najmudin, C. A. J. Palmer, K. Ta Phuoc, W. Schumaker, L. O. Silva, J. Vieira, V. Yanovsky, and A. G. R. Thomas, Phys. Rev. ST Accel. Beams 15 (2), 021302 (2012).

G. R. Plateau, C. G. R. Geddes, D. B. Thorn, M. Chen, C. Benedetti, E. Esarey, A. J. Gonsalves, N. H. Matlis, K. Nakamura, C. B. Schroeder, S. Shiraishi, T. Sokollik, J. van Tilborg, Cs Toth, S. Trotsenko, T. S. Kim, M. Battaglia, Th Stöhlker, and W. P. Leemans, Phys. Rev. Lett. 109 (6), 064802 (2012).

C. Fourment, N. Arazam, C. Bonte, T. Caillaud, D. Descamps, F. Dorchies, M. Harmand, S. Hulin, S. Petit, and J. J. Santos, Rev. Sci. Instrum. 80 (8), 083505 (2009). 

C. Fourment, V. Malka, S. Sebban, A. Rousse, and J. C. Kieffer, New J. Phys. 13 (3), 033017 (2011).

Michael Schnell, Alexander Sävert, Ingo Uschmann, Oliver Jansen, Malte Christoph Kaluza, and Christian Spielmann, J. Plasma Phys. 81 (4) (2015).

Kazuo A. Tanaka, Toshinori Yabuuchi, Takashi Sato, Ryosuke Kodama, Yoneyoshi Kitagawa, Teruyoshi Takahashi, Toshiji Ikeda, Yoshihide Honda, and Shuuichi Okuda, Rev. Sci. Instrum. 76 (1), 013507 (2004).

C. H. Yu, J. S. Liu, W. T. Wang, W. T. Li, R. Qi, Z. J. Zhang, Z. Y. Qin, J. Q. Liu, M. Fang, K. Feng, Y. Wu, C. Wang, Y. Xu, Y. X. Leng, C. Q. Xia, R. X. Li, and Z. Z. Xu, arXiv:1706.01033 [physics.plasm-ph].

A. G. R. Thomas, Phys. Rev. ST Accel. Beams 13 (2), 020702 (2010).

M. Chen, E. Esarey, C. G. R. Geddes, C. B. Schroeder, G. R. Plateau, S. S. Bulanov, S. Rykovanov, and W. P. Leemans, Phys. Rev. ST Accel. Beams 16 (3), 030701 (2013).

S. Kiselev, A. Pukhov, and I. Kostyukov, Phys. Rev. Lett. 93 (13), 135004 (2004).

Chet Nieter and John R. Cary, J. Comput. Phys. 196 (2), 448 (2004).

Z. J. Zhang, W. T. Li, J. S. Liu, W. T. Wang, C. H. Yu, Y. Tian, Kazuhisa Nakajima, A. H. Deng, R. Qi, C. Wang, Z. Y. Qin, M. Fang, J. Q. Liu, C. Q. Xia, R. X. Li, and Z. Z. Xu, Phys. Plasmas 23 (5), 053106 (2016).

A. H. Deng, J. S. Liu, K. Nakajima, C. Q. Xia, W. T. Wang, W. T. Li, H. Y. Lu, H. Zhang, J. J. Ju, Y. Tian, Ch Wang, R. X. Li, and Z. Z. Xu, Phys. Plasmas 19 (2), 023105 (2012).

Y. Ma, L. M. Chen, N. A. M. Hafz, D. Z. Li, K. Huang, W. C. Yan, J. Dunn, Z. M. Sheng, and J. Zhang, Appl. Phys. Lett. 105 (16), 161110 (2014). 